\title{
Regional cosmic ray induced ionization and geomagnetic field changes
}

\author{
G. A. Kovaltsov ${ }^{1}$ and I. G. Usoskin ${ }^{2}$ \\ ${ }^{1}$ Ioffe Physical-Technical Institute, St. Petersburg, Russia \\ ${ }^{2}$ Sodankylä Geophysical Observatory (Oulu Unit), University of Oulu, Finland
}

Received: 16 October 2006 - Revised: 4 December 2006 - Accepted: 27 February 2007 - Published: 13 August 2007

\begin{abstract}
Cosmic ray induced ionization (CRII) is an important factor of outer space influences on atmospheric properties. Variations of CRII are caused by two different processes - solar activity variations, which modulate the cosmic ray flux in interplanetary space, and changes of the geomagnetic field, which affects the cosmic ray access to Earth. Migration of the geomagnetic dipole axis may greatly alter CRII in some regions on a time scale of centuries and longer. Here we present a study of CRII regional effects of the geomagnetic field changes during the last millennium for two regions: Europe and the Far East. We show that regional effects of the migration of the geomagnetic dipole axis may overcome global changes due to solar activity variations.
\end{abstract}

\section{Introduction}

Presently, there are numerous arguments suggesting that solar magnetic variability affects the global climate in different aspects and on different time scales (see, e.g., de Jager, 2005; Haigh, Lockwood and Giampapa, 2005; Versteegh, 2005). An important factor affecting the terrestrial environment is the flux of cosmic rays permanently impinging on the Earth. In particular, galactic cosmic rays (CR) form the dominant source of ionization in the atmosphere, especially the troposphere. Variations of the cosmic ray flux lead to significant changes in the cosmic ray induced ionization (CRII), which in turn may modulate processes related to the formation of clouds (e.g. Ney, 1959; Svensmark, 2000; Carslaw et al., 2002). There is much evidence for a significant correlation between the cosmic ray flux and low cloud cover on a global scale during the last 20 years (e.g., Marsh and Svensmark, 2000; Pallé Bago and Butler, 2000). On the other hand, the relation between CRII and cloud coverage is not uniform and

Correspondence to: I. G. Usoskin

(ilya.usoskin@oulu.fi) has a clear geographical pattern - strong correlations are limited to several large areas (e.g., the Northern Atlantic and Europe, the Far East, and the circum-Antarctic region), where cloud formation conditions may be more sensitive to CRII variations (Pallé et al., 2004; Usoskin et al., 2004a). Therefore, changes of CRII in these regions can produce a larger effect on the cloud cover and thus on the climate than global CRII variations.

The cosmic ray flux, and the ensuing CRII, are modulated by several processes. The most obvious is solar modulation which leads to the well-known 11-year cycle in CR intensity but also causes long-term variations (e.g., Usoskin et al., 2003; Solanki et al., 2004). On the other hand, local CRII is defined by the flux of CR which can access a given location on the Earth. Variations of the local CR flux are caused not only by the overall modulation of CR by solar activity but also by changes of the local geomagnetic rigidity cutoff (the minimum rigidity of $\mathrm{CR}$ needed to access the location). The latter is defined by both the global dipole moment of the geomagnetic field and by the geomagnetic latitude of the site (details are discussed in Sect. 2), which vary with time because of the magnetic axis migration (Kudela and Bobik, 2004; Shea and Smart, 2004a,b).

Most of the earlier studies consider possible climatic effects of either solar activity or cosmic ray flux at the Earth's orbit (e.g., Shaviv, 2005; Usoskin et al., 2005a). However, the local variations of CRII in some locations may be dominated on long time scales, centennial and longer, not by these global processes but by geomagnetic field changes. In this paper we consider such CRII variations in two regions in the Northern Hemisphere with the highest correlation between cloud formation and CRII - Europe and the Far East - and estimate the role of geomagnetic field variations on atmospheric ionization for the last millennium. 


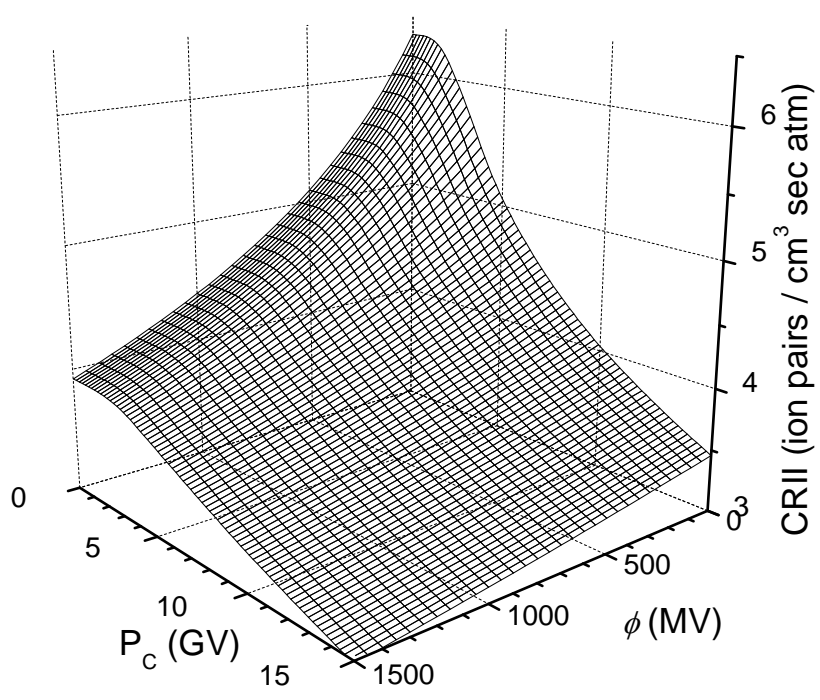

Fig. 1. Cosmic ray induced ionization of the atmosphere at an altitude of $3 \mathrm{~km}$ (depth $700 \mathrm{~g} / \mathrm{cm}^{-2}$ ) as a function of the geomagnetic cutoff rigidity $P_{c}$ and modulation potential $\phi$.

\section{Cosmic Ray Induced Ionization}

The ionization rate in the atmosphere can be represented in the following form:

$Q\left(x, \phi, P_{c}\right)=\sum_{i} \int_{T_{\mathrm{c}, i}}^{\infty} J_{i}(T, \phi) Y_{i}(x, T) d T$,

where the summation is performed over different $i$ th species of CR (protons, $\alpha$-particles, heavier species), $Y_{i}(x, T)$ is the ionization yield function (the number of ion pairs produced at altitude $x$ in the atmosphere by one CR particle of the $i$ th type with kinetic energy $T$ - see Usoskin et al. (2004b, 2006a)), $J_{i}(T, \phi)$ is the differential energy spectrum of the $i$ th species of GCR that is defined by the modulation potential $\phi$ which depends on the solar activity level (see CaballeroLopez and Moraal, 2004; Usoskin et al., 2005b). Integration is over the kinetic energy $T$ above $T_{\mathrm{c}, i}$, which is kinetic energy corresponding to the local geomagnetic rigidity cutoff $P_{\mathrm{c}}$. The value of $P_{\mathrm{c}}$ can be approximately computed using Störmer's approximation (Elsasser et al., 1956):

$P_{\mathrm{c}} \approx 1.9 \times M \cos ^{4} \lambda_{\mathrm{G}}$,

where $P_{\mathrm{c}}$ is the vertical geomagnetic cutoff in $\mathrm{GV}, M$ is the dipole moment of the geomagnetic field expressed in $10^{25}$ Gauss $\mathrm{cm}^{3}$, and $\lambda_{\mathrm{G}}$ is the local geomagnetic latitude. $\lambda_{\mathrm{G}}$ can be calculated from local geographical coordinates $(\lambda ; \phi)$ and the coordinates of the magnetic pole $\left(\lambda_{\mathrm{P}} ; \phi_{\mathrm{P}}\right)$

$\sin \lambda_{\mathrm{G}}=\sin \lambda_{\mathrm{P}} \times \sin \lambda+\cos \lambda_{\mathrm{P}} \times \cos \lambda \times \cos \left(\phi_{\mathrm{P}}-\phi\right)(3)$

Thus one can see from Eq. 1 that the local CRII depends on both the level of solar activity (quantified via the modulation potential $\phi$ ) and on local parameters of the geomagnetic field
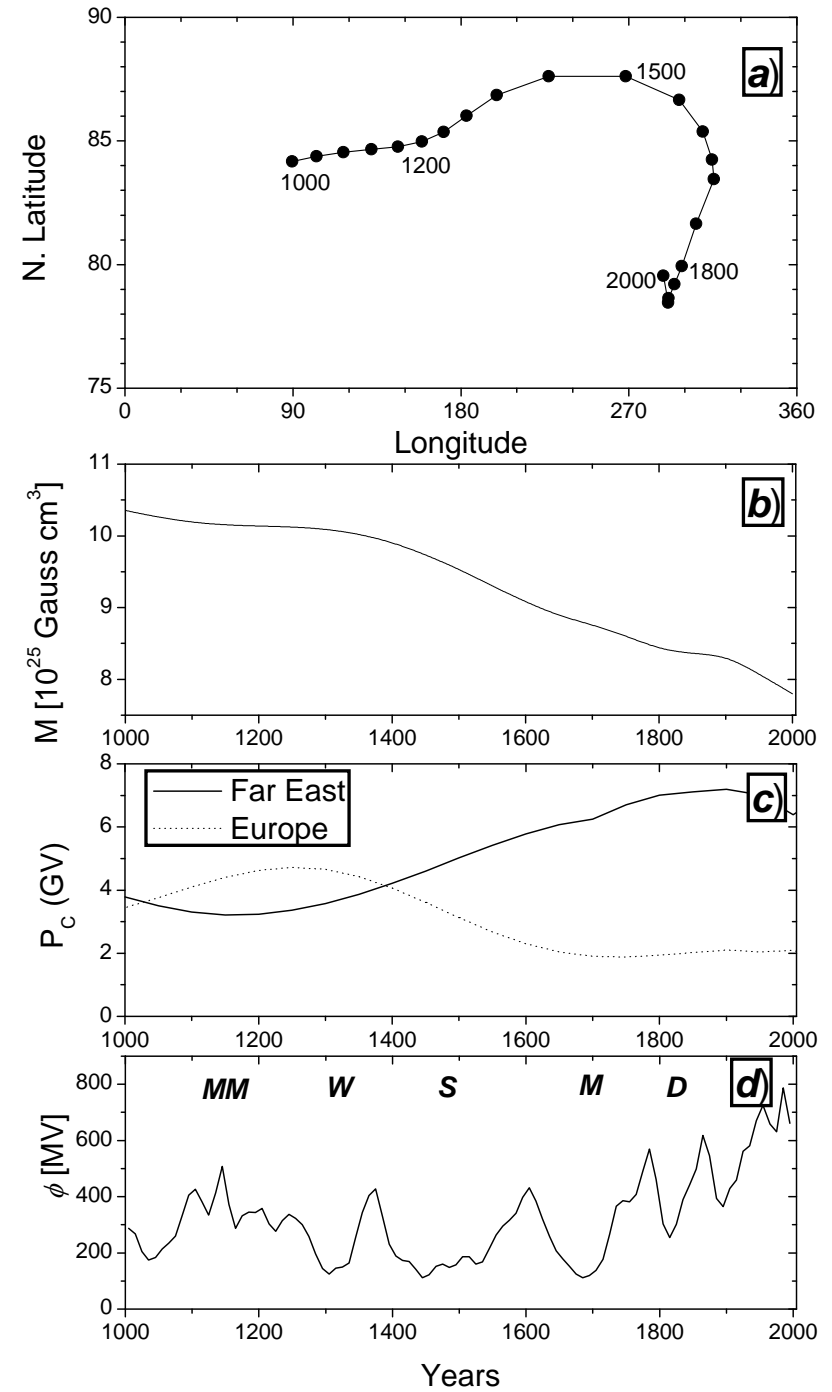

Fig. 2. Changes during the last millennium: (a) Migration of the geomagnetic pole; (b) Dipole moment; (c) Geomagnetic cutoff rigidity in Europe (geographical $50^{\circ} \mathrm{N} 0^{\circ} \mathrm{E}$ ) and Far East (geographical $45^{\circ} \mathrm{N} 140^{\circ} \mathrm{E}$ ); (d) Modulation potential (decadal averages). Letters denote the Medieval maximum (MM) as well as the Wolf (W), Spörer (S), Maunder (M) and Dalton (D) minima of solar activity.

(quantified via the geomagnetic rigidity cutoff $P_{\mathrm{c}}$ ). The dependence of CRII (computed by the method of Usoskin et al. (2006a)) on the values of $P_{\mathrm{c}}$ and $\phi$ is shown in Fig. 1 for an altitude of about $3 \mathrm{~km}$ (atmospheric depth $700 \mathrm{~g} / \mathrm{cm}^{2}$ ). The range shown in the figure covers a range of realistic values of $\phi$ (from the Maunder minimum up to very high solar activity with $\phi \sim 1500 \mathrm{MV}$ ) and $P_{\mathrm{c}}$ (from poles up to equatorial cutoff of $15 \mathrm{GV})$. 


\section{Geomagnetic field and solar activity changes during the last millennium}

The geomagnetic field changes in time, due to the varying dipole moment $M$ and to geomagnetic pole migration. The latter leads to a varying geomagnetic latitude $\lambda_{\mathrm{G}}$ for a given geographical location. The dipole moment was gradually decreasing during the last millennium (see Fig. 2b) by nearly $1 / 3$ of its value. Here we use data of the geomagnetic reconstructions by Bloxham and Jackson (1992) and Hongre et al. (1998) before 1900 and the IGRG/DGRF model after 1900. Changes of the dipole axis were quite rapid during the last millennium (see Fig. 2a), so that the northern (magnetically southern) pole migrated from the Severnaya Zemlya archipelago circa $1000 \mathrm{AD}$ to Northern Greenland nowadays, i.e., by around $180^{\circ}$ in geographical longitude.

Both $M$ and $\lambda_{\mathrm{G}}$ define the local geomagnetic cutoff (Eq. 2). The pole migration may greatly modify the value of $P_{\mathrm{c}}$ in some locations, particularly in mid-latitudes, leading to significant variations of $P_{\mathrm{c}}$ and thus CRII in these regions.

Solar activity was also changing greatly during the last millennium affecting the variability of the cosmic ray flux, and thus CRII. Fig. 2d shows time profile of the heliospheric modulation $\phi$ (Solanki et al., 2004) reconstructed from data on the cosmogenic isotope ${ }^{14} \mathrm{C}$ measured in tree rings. Note that ${ }^{14} \mathrm{C}$ is globally mixed in the atmosphere before deposition in a natural archive and therefore is affected by the dipole moment (which has been taken into account by the reconstruction of $\phi$ ) but is insensitive to the dipole axis migration. It is notable that the solar activity was significantly lower in the past than during the last few decades (Usoskin et al., 2003; Solanki et al., 2004), which implies that global CRII would have been higher in the past.

\section{Effects in CRII}

In order to study the effects of geomagnetic-field changes, we consider in detail two regions: the European region (including the East-North Atlantic affected by the Gulf stream) with its centre at geographical $50^{\circ} \mathrm{N} 0^{\circ} \mathrm{E}$ and the Far East region with its centre at $45^{\circ} \mathrm{N} 140^{\circ} \mathrm{E}$. We note that these regions correspond to areas where the CRII may strongly affect low cloud formation (Usoskin et al., 2006b; Voiculescu et al., 2006). Therefore, variations of the CRII in these regions may be relevant for climate changes. Long-term changes of the geomagnetic cutoff in these regions are shown in Fig. 2c. One can see that these changes are quite dramatic, by more than a factor of two: $P_{\mathrm{c}}$ changed between 1.9 and $4.7 \mathrm{GV}$ in Europe and between 3.2 and 7.2 GV in the Far East. The corresponding changes in the geomagnetic latitude were between $45^{\circ}$ and $55^{\circ}$ and $35^{\circ}$ and $50^{\circ}$, respectively, but $P_{\mathrm{c}}$ changes include also changes of $M$.

Using the computed changes in the local geomagnetic rigidity cutoff $P_{\mathrm{c}}$ (see Fig. 2c) and the reconstructed solar

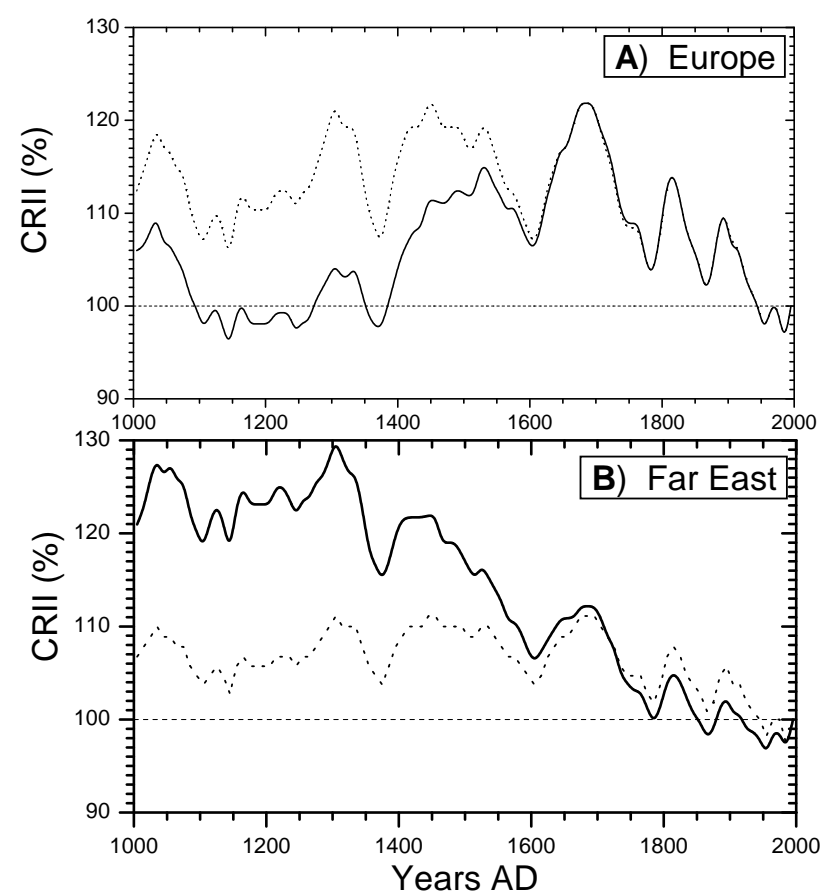

Fig. 3. Decadal averaged cosmic ray induced ionization (solid curves) at $3 \mathrm{~km}$ altitude $\left(700 \mathrm{~g} / \mathrm{cm}^{-2}\right.$ depth) in Europe (geographical $50^{\circ} \mathrm{N} 0^{\circ} \mathrm{E}$ ) and the Far East (geographical $45^{\circ} \mathrm{N} 140^{\circ} \mathrm{E}$ ). Dotted curves correspond to hypothetical variations if there were no changes in the geomagnetic field, i.e. changes are attributed to only solar activity variations. All curves were normalized to the period 1990-2000 (4.82 and 4.13 ion pairs $\mathrm{cm}^{-3} \mathrm{~s}^{-1} \mathrm{~atm}^{-1}$ for Europe and the Far East, respectively), which is depicted by the dashed line.

modulation of galactic CR (Fig. 2d), and applying the CRII model (Fig. 1), we have calculated the expected changes in CRII in the two selected regions, which are shown as solid curves in Fig. 3. These changes include both solar activity and geomagnetic field changes. In order to disentangle solar and geomagnetic field changes in the CRII variations, we have also computed CRII changes caused only by solar activity changes (dotted line in Fig. 3), i.e., assuming that the geomagnetic field did not change but remained the same as now.

In the European region, the absolute maximum of the CRII occurred during the Maunder minimum with the ionization rate being 20\% higher than nowadays (Fig. 3a). During the Medieval maximum (ca. 1200 AD) the CRII level was very similar to the modern level. This pattern resembles global climatic reconstructions: Medieval warming followed by the Little Ice age and then the Modern warming (see, e.g., Eddy, 1977). Here we note that it still a matter of intense debate whether these pronounced climatic features were regional (related to European regions) or global. On the other hand, these CRII variations were largely affected by geomagnetic changes, e.g., the CRII would have been $10 \%$ higher 800 
years ago than now if there were no changes in the geomagnetic field. This implies that the long-term changes in CRII in Europe were mostly defined by the migrating geomagnetic pole before $1500 \mathrm{AD}$ rather than by solar activity variations.

The situation is quite different in the Far East. The changes of CRII would be small, within $10 \%$, if the geomagnetic field was the same as now (Fig. 3b). However, the full span of CRII changes is estimated to be as large as $30 \%$ between ca. 1300 and the modern epoch. Moreover, the CRII rate was 25\% higher during the Medieval maximum than now, due to changes in the geomagnetic rigidity cutoff (see Fig. 2c). This is contrary to the pattern for the European region.

From this study we can conclude that local CRII variations may be largely affected by geomagnetic field changes, and not only by the global modulation of the CR flux by solar activity.

\section{Conclusions}

The Earth's climate is not formed/modulated uniformly over the globe, but is mostly determined by conditions in some specific key regions, which in turn affect larger regions or global climate features. Thus, the global climate can be affected via changes of not only global atmospheric or ocean parameters, but also via local changes if they are related to such key regions. Here we have considered an important atmospheric parameter, the cosmic ray induced ionization, in two regions in the Northern Hemisphere - the European region and the Far East region - during the last millennium. We have shown that CRII variations in these two regions are dominated by changes caused by the migration of the geomagnetic pole, which exceed those variations due to solar activity changes.

We note that the migration of the magnetic pole during the last millennium, which caused significant effects in cosmic ray induced ionization variations in some regions, was not exceptional. Actually, it can be regarded as a minor excursion (about $2000 \mathrm{~km}$ or $18^{\circ}$ of a great circle during the millennium). There is evidence for more dramatic excursions of the geomagnetic axis, even for historical times. For example, the magnetic pole could have migrated for more that $90^{\circ}$ of a great circle during the so-called "Sterno-Etrussia" geomagnetic excursion around 700 BC (Dergachev et al., 2004). The corresponding changes in local CRII must then be dramatic and may result in strong regional effects.

It is important to realize that temporal variations of the insolation at any location on Earth are defined by the variability of the solar irradiance and thus are synchronous all over the globe. On the other hand, regional changes in CRII can be affected not only by the varying flux of galactic cosmic rays but also by geomagnetic field changes, that may exhibit very particular regional features. Accordingly, studies of regional effects may shed new light on debates concerning a particular mechanism responsible for the solar variability- climate link - cosmic rays or solar radiance (Haigh, 1996; Tinsley, 1996; Yu, 2002; Kristjansson et al., 2004; Carslaw et al., 2002; Haigh, Lockwood and Giampapa, 2005).

We conclude that local effects in variations of the cosmic ray flux, which may dominate over the globally averaged changes in some locations, should be taken into account in long-term studies of solar-terrestrial relations.

Acknowledgements. Suomalainen Tiedeakatemia (Vilho, Yrjö and Kalle Väisälä Foundation), the Academy of Finland, and Russian Academy of Sciences (Project \#30) are thanked for financial support.

Edited by: N. Crosby and M. Rycroft

Reviewed by: two anonymous referees

\section{References}

Bloxham, J. and Jackson, A.: Time-dependent mapping of the magnetic field at the core-mantle boundary, J. Geophys. Res., 97, 19537-19563, 1992.

Caballero-Lopez, R. A. and Moraal, H.: Limitations of the force field equation to describe cosmic ray modulation, J. Geophys. Res., 109(A1), A01101, doi:10.1029/2003JA010098, 2004.

Carslaw, K. S., Harrison, R. G., and Kirkby, J.: Cosmic rays, clouds, and climate, Science, 298, 1732-1737, 2002

de Jager, C.: Solar forcing of climate. 1: Solar variability, Space Sci. Rev., 120, 197-241, 2005.

Dergachev, V. A., Raspopov, O. M., van Geel, B., and Zaitseva, G. I.: The 'Sterno-Etrussia' geomagnetic excursion around $2700 \mathrm{BP}$ and changes of solar activity, cosmic ray intensity, and climate, Radiocarbon, 46, 661-681, 2004.

Eddy, J. A.: Climate and changing Sun, Climatic Change, 1, 173 190, 1977.

Elsasser, W., Ney, E. P., and Winckler, J. R.: Cosmic-ray intensity and geomagnetism, Nature, 178, 1226-1227, 1956.

Haigh, J. D.: The impact of solar variability on climate, Science, 272(5264), 981-984, 1996.

Haigh, J. D., Lockwood, M., and Giampapa, M. S.: The Sun, solar analogs and the climate, Springer-Verlag, Berlin Heidelberg, 424 pp., 2005.

Hongre, L., Hulot, G., and Khokhlov, A.: An analysis of the geomagnetic field over the past 2000 years, Phys. Earth Planet. Inter. 106, 311-335, 1998.

Kristjansson, J. E., Kristiansen, J., and Kaas, E.: Solar activity, cosmic rays, clouds and climate - an update, Adv. Space Res., 34 407-415, 2004.

Kudela, K. and Bobik, P.: Long-term variations of geomagnetic rigidity cutoffs, Solar Phys., 224, 423-431, 2004.

Marsh N. and Svensmark, H.: Low Cloud Properties Influenced by Cosmic Rays, Phys. Rev. Lett., 85, 5004-5007, 2000.

Ney, E. P.: Cosmic radiation and the weather, Nature, 183, 451-452, 1959.

Pallé Bago, E. and Butler, C. J.: The influence of cosmic rays on terrestrial clouds and global warming, Astron. Geophys., 41(4), $18-22,2000$

Pallé, E., Butler, C. J., and O'Brien, K.: The possible connection between ionization in the atmosphere by cosmic rays and low level clouds, J. Atm. Solar-Terr. Phys., 66, 1779-1790, 2004. 
Shaviv, N. J.: On climate response to changes in the cosmic ray flux and radiative budget, J. Geophys. Res., 110, A08105, doi:10.1029/2004JA010866, 2005.

Shea, M. A. and Smart, D. F.: Preliminary study of cosmic rays, geomagnetic field changes and possible climate changes, Adv. Space. Res., 34(2), 420-425, 2004a.

Shea, M. A. and Smart, D. F.: The use of geophysical data in studies of the historical solar-terrestrial environment, Solar Phys., 224, 483-493, 2004b.

Solanki, S. K., Usoskin, I. G., Kromer, B., et al.: An unusually active Sun during recent decades compared to the previous 11,000 years, Nature, 431, 1084-1087, 2004.

Svensmark, H.: Cosmic rays and Earth's climate, Space Sci. Rev., 93, 175-185, 2000.

Tinsley, B. A.: Correlations of atmospheric dynamics with solar wind-induced changes of air-earth current density into cloud tops, J. Geophys. Res., 101, 29701-29714, 1996.

Usoskin, I. G., Solanki, S. K., Schuessler, M., Mursula, K., and Alanko, K.: Millennium-Scale Sunspot Number Reconstruction: Evidence for an Unusually Active Sun since the 1940s, Phys. Rev. Lett., 91(21), 211101, doi:10.1103/PhysRevLett.91.211101, 2003.

Usoskin, I. G., Marsh, N., Kovaltsov, G. A., et al.: Latitudinal dependence of low cloud amount on cosmic ray induced ionization, Geophys. Res. Lett., 31(16), L16109, doi:10.1029/2004GL019507, 2004a.

Usoskin, I. G., Gladysheva, O. G., and Kovaltsov, G. A.: Cosmic ray induced ionization in the atmosphere: Spatial and temporal changes, J. Atmos. Solar-Terr. Phys., 66(18), 1791-1796, $2004 \mathrm{~b}$.
Usoskin, I. G., Schüssler, M., Solanki, S., and Mursula, K.: Solar activity, cosmic rays and Earth's temperature: A millennium-scale comparison, J. Geophys. Res., 110, A10102, doi:10.1029/2004JA010946, 2005a.

Usoskin, I. G., Alanko-Huotari, K., Kovaltsov, G. A., and Mursula, K.: Heliospheric modulation of cosmic rays: Monthly reconstruction for 1951-2004, J. Geophys. Res., 110, A12108, doi:10.1029/2005JA011250, 2005b.

Usoskin, I. G. and Kovaltsov, G. A.: Cosmic Ray Induced Ionization in the Atmosphere: Full Modeling and Practical Applications, J. Geophys. Res., 111, D21206, doi:10.1029/2006JD007150, 2006a.

Usoskin, I. G., Voiculescu, M., Kovaltsov, G. A., and Mursula, K.: Correlation between clouds at different altitudes and solar activity: Fact or Artifact?, J. Atmos. Solar-Terr. Phys., 68, 2164 2172, 2006b.

Versteegh, G. J. M.: Solar forcing of climate. 2: Evidence from the past, Space Sci. Rev., 120, 243-286, 2005.

Voiculescu, M., Usoskin, I. G., and Mursula, K.: Different response of clouds at the solar input, Geophys. Res. Lett., 2006, 33, L21802, doi:10.1029/2006GL027820, 2006.

Yu, F.: Altitude variations of cosmic ray induced production of aerosols: Implications for global cloudiness and climate, J. Geophys. Res., 107(A7), 1118, doi:10.1029/2001JA000248, 2002. 